IAC-04-I.2.02

\title{
ON THE STRUCTURAL OPTIMIZATION FOR SPACE APPLICATIONS USING THE MIN-MAX TECHNIQUE
}

\author{
Alfredo R. de Faria \\ Instituto Tecnológico de Aeronáutica - ITA, São José dos Campos, Brazil \\ arfaria@ita.br \\ Leandro Cardozo \\ Fibraforte Engenharia Indústria e Comércio Ltda, São José dos Campos, Brazil \\ leandroinpe@yahoo.com.br \\ Ijar M. Fonseca \\ Instituto Nacional de Pesquisas Espaciais - INPE, São José dos Campos, Brazil \\ ijar@dem.inpe.br
}

\begin{abstract}
Optimization plays a very important role in space applications since mass and structural flexibility are critical requirements for space missions, i.e., the structure should be as lightweight and stiff as possible. As a contribution to this area this paper investigates compliance optimization of structures under multiple load cases. The problem can be solved through a multi-criterion optimization where the load cases associated with each and every loading configuration are treated as components of multi-objective function vector. However, numerical evaluation is not an easy task because it sometimes involves a very intensive computational effort. Alternatively, the multi-objective optimization problem can be re-formulated using a min-max strategy that does not require simultaneous consideration of all the load cases as components of multi-objective function vector. Instead, this formulation shows that, for compliance optimization purposes, it is sufficient to consider only those loads, which define the convex hull of the applicable load set, i.e., the selected set of loads which will effectively lead to the optimum design. Through the min-max formulation the number of load cases involved in the design procedure is drastically reduced. The efficiency of the proposed technique is illustrated by one example consisting of a variable thickness beam subjected to uncertain loadings.
\end{abstract}




\section{INTRODUCTION}

Aircraft wings and fuselage, solar panels of satellites, engine pistons and solid propellant rocket engines are some examples of aerospace structural components that can be considered critical because they are submitted to many - hundreds or even thousands - of load cases. And it's known that, during the operation of the structural component, the loading configuration may change.

Another worrisome aspect structural design is the consideration of uniform loading distribution. Most design manuals bring tables and graphs based on this consideration, but hardly ever all loading configurations are perfectly uniformly distributed.

When a structural optimization is performed to find a lightweight and stiff structural component, the optimization toll often considers only one or few load cases. Hence, the optimum designs obtained may become sensitive or even vulnerable to loading variation. This problem can be solved or alleviated by a multi-criterion optimization formulation where the load cases associated with each and every loading configuration are treated as components of a multi-objective function vector [1]. However, numerical evaluation of such method is not an easy task and involves intensive computational procedures.

Another optimization technique is the uncertain load approach. Using probabilistic methods, the uncertain approach is more refined and conservative than the approach described above. This method describes a load space through probability density distributions. The loads have distinct probabilities of occurrence. However, the most difficult problem is to select the load case with the highest probability of occurrence and to know what probability distribution is that.

Alternatively, the multi-objective optimization problem can be re-formulated using a min-max strategy that requires neither simultaneous consideration of all the load cases as components of a multi-objective function vector nor probability density distributions. Since there are hundreds or even thousands of load cases involved in practical structural designs, the min-max methodology adopted is based on a well- defined loading space that contains all the possible load cases and all convex combinations of them. The optimization performed delivers two results: the optimal design and the worst load cases.

This methodology proposed is based on convex modeling and the considerations of an admissible loading space. All elements of this loading space have equal probability of occurrence. This approach does not depend on the probability distributions but on the extremal properties of the loading space chosen. The optimum design refers to the most severe load belonged to an admissible load set. This technique assures that the optimum design provided by this elected load is conservative and also optimum to the others because were considered less harmful to the optimal design.

\section{MULTI-CRITERIA OPTIMIZATION}

There are optimization problems that require not only one objective function but several of them, each objective function representing a different feature of performance index. For example, it is sometimes desired to decrease the mass of a satellite solar panel and also increase its global fundamental frequencies.

The components of the multi-objective function vector may be conflicting. It is usually observed that when a given component is improved with respect to the some design variables the same design variables impair other components. So, the strategy optimization must be able to deal with many design criteria involved and with the possible competition among them.

A general multi-objective function optimization problem may be stated as in equation (1).

$$
\min _{\mathrm{x} \in G}\left\{\begin{array}{c}
f_{1}(\mathrm{x}) \\
f_{2}(\mathrm{x}) \\
\ldots \\
f_{l-1}(\mathrm{x}) \\
f_{l}(\mathrm{x})
\end{array}\right\},
$$

where $G$ represents the feasible design space defined by equality and inequality constraints. In practical problems, there is not 
an optimum point $\mathbf{x}^{*}$ that minimizes all the functions $f_{i}$ simultaneously. Thus, it is desired to find a particular design $\hat{\mathbf{x}}$ that is better than another design $\overline{\mathbf{x}}$ is some sense.

The idea sorting designs according to some criterion relates to the concept of dominance. It can be mathematically stated considering that $\hat{\mathbf{x}}$ dominates over another design $\overline{\mathbf{x}}$ when $f_{i}(\hat{\mathbf{x}}) \leq f_{i}(\overline{\mathbf{x}})$ for all $i \in[1,2, \ldots, l]$ and $f_{j}(\hat{\mathbf{x}})<f_{j}(\overline{\mathbf{x}})$ for some $j \in[1,2, \ldots, l]$. A design $\widehat{\mathbf{x}}$ is said to be a Pareto design if and only if there does not exist a design $\mathbf{x} \in G$ that dominates over $\hat{\mathbf{x}}$. If there is a single design $\mathbf{x}^{*}$ that minimizes all the functions $f_{i}$, this is the only Pareto design for that multicriteria optimization problem. However, as stated above, it is common to have a multitude of Pareto designs and the engineer must elect one of them as the effective optimal design.

\section{THE MIN-MAX TECHNIQUE}

An alternative formulation to the multicriteria optimization problem is to minimize the maximum $f_{i}(\mathbf{x})$ for all $i \in[1, \ldots, l]$. The minimax approach simultaneously minimizes $f_{i}(\mathbf{x})$ with respect to $\mathbf{x} \in G$ and maximizes it with respect to $i \in[1, \ldots, l]$. It can be stated as in the equation (2).

$$
\min _{\mathbf{x} \in G} \max _{i \in[1, \ldots, l]} f_{i}(\mathbf{x})
$$

There are two types of design variable in equation (2): the first one is the continuous design variable $\mathbf{x}$ and the second one is the discrete design variable $i$. Another way to state minimax optimization problem with only continuous design variables is presented in equation (3).

$$
\min _{\mathbf{x} \in X} \max _{\mathbf{y} \in Y} f(\mathbf{x}, \mathbf{y})
$$

Cherkaev and Cherkaeva [2] applied the minimax formulation in structural optimization. They studied the sensitivity of the optimal designs to variations on the applied load. Their idea was to minimize the design strain energy under the most harmful load case elected among a defined set of applied load.
This section will deal with the linear problem of minimum strain energy stored in given structure when it is subjected to uncertain loads. The strain energy, also known as compliance, measures the structure flexibility. So it is possible to evaluate the structure deformations, stresses and strains and also estimate the natural frequencies using Rayleigh's quotient $[3,4]$.

The goal of this present work is to apply the minimax method on the strain energy minimization of structures under uncertain loads. The uncertain loads belong to a load space where any element is referred by $\mathbf{f}$. When $\mathbf{f}$ is fixed, i.e., one particular load is selected, the strain energy can be stated as in equation (4).

$$
\min _{\mathbf{h} \in H} C(\mathbf{h}, \mathbf{f})
$$

where $H$ is the design space where all constraints on $\mathbf{h}$ are satisfied. The design variable $\mathbf{h}$ may be related to the thickness distribution of a component, fiber angle orientation and number of plies in the case of composite structure, position of stiffeners, etc. But, $\mathbf{f}$ is not a fixed load because it is assumed uncertain. Therefore, the statement of the multi-objective optimization approach may be given as in equation (5).

$$
\min _{\mathbf{h} \in H}\left\{\begin{array}{c}
C_{1}\left(\mathbf{h}, \mathbf{f}_{1}\right) \\
C_{2}\left(\mathbf{h}, \mathbf{f}_{2}\right) \\
\ldots \\
C_{l-1}\left(\mathbf{h}, \mathbf{f}_{l-1}\right) \\
C_{l}\left(\mathbf{h}, \mathbf{f}_{l}\right)
\end{array}\right\}
$$

The problem in equation (5) must be solved using a multi-criterion approach. However, when the min-max strategy is applied, the problem can be reformulated as in the equation (6).

$$
\min _{\mathbf{h} \in H} \max _{\mathbf{f} \in F} C(\mathbf{h}, \mathbf{f})
$$

where $F$ is the load space that contains all the loads $\mathbf{f}_{1}, \mathbf{f}_{2}, \ldots, \mathbf{f}_{l}$.

The optimization presented in equation (6) is bilevel. It gives the solution of the best design in terms of $\mathbf{h}$ and the worst load case referred 
to as $\mathbf{f}$. Equation (6) can also be expressed by equation (7).

$\min _{\mathbf{h} \in H} \max _{\mathbf{f} \in F} C(\mathbf{h}, \mathbf{f})=\min _{\mathbf{h} \in H} \phi(\mathbf{h}), \quad \phi(\mathbf{h})=\max _{\mathbf{f} \in F} C(\mathbf{h}, \mathbf{f})$ (7)

Equation (7) maximizes the objective function $\phi$ with respect to the uncertain loads $\mathbf{f}$, and after, minimizes it with respect to $\mathbf{h}$. This approach assures the optimal design can withstand loading perturbations because any variation of $\mathbf{f}$ within the admissible load space $F$, necessarily leads to lower and not harmful strain energy. This is because the "max" part of the bilevel optimization already identified the most dangerous loading configuration.

\section{THEORETICAL ASPECTS}

Basic concepts of convex sets, convex hulls and convex functions will be given in order to facilitate explanation of the coming optimization strategy.

Consider a set whose elements is a collection composed by $\mathbf{x}_{1}, \quad \mathbf{x}_{2}, \ldots, \quad \mathbf{x}_{\mathrm{n}}$. The point $\mathbf{x}=\sum_{i=1}^{n} \xi_{i} \mathbf{x}_{i}$ is said to be a convex combination of $\mathbf{x}_{1}, \quad \mathbf{x}_{2}, \quad \ldots, \quad \mathbf{x}_{n}$ when $\sum_{i=1}^{n} \xi_{i}=\mathbf{1}$ and $\xi_{i} \geq 0$ for all $i \in[1, \ldots, n]$.

A function $f(\mathbf{x})$ is convex when $f\left((1-\theta) \mathbf{x}_{1}+\theta\right.$ $\left.\mathbf{x}_{2}\right) \leq(1-\theta) f\left(\mathbf{x}_{1}\right)+\theta f\left(\mathbf{x}_{2}\right)$ for $0 \leq \theta \leq 1$ and for any $\mathbf{x}_{1}$ and $\mathbf{x}_{2}$. Conversely, a function is concave when when $f\left((1-\theta) \mathbf{x}_{1}+\theta \mathbf{x}_{2}\right) \geq(1-\theta)$ $f\left(\mathbf{x}_{1}\right)+\theta f\left(\mathbf{x}_{2}\right)$ for $0 \leq \theta \leq 1$ and for any $\mathbf{x}_{1}$ and $\mathbf{x}_{2}$.

A set is said convex when, for any elements $A$ and $B$ in the set, the element $P=(1-\theta) A+\theta$ $B$ is also in the set, where $0 \leq \theta \leq 1$.

The conclusion drawn from the definitions of convex combination and convex set is that any point within the interior of the convex set can be written as a convex combination of the points on its boundary. Based on this observation, the convex hull $\Omega_{\text {hull }}$ of a given set $\Omega$ can be defined as the smallest subset of $\Omega$ such that each and every element in $\Omega$ can be written as a convex combination of the elements in $\Omega_{\text {hull. }}$. It can be concluded from this definition that the convex hull of any polyhedron is the set of points of its vertices (Fig. 1).

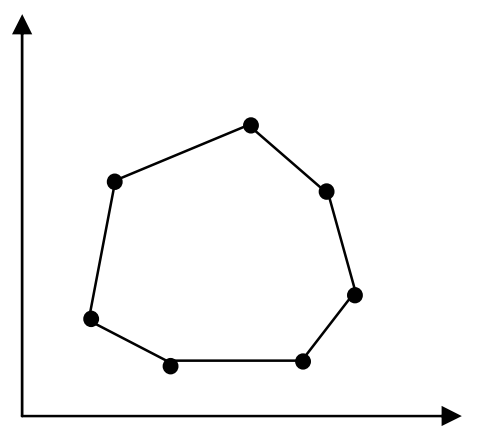

Figure 1: Convex hull of a convex polygon

Based on the above principles, it can be shown that the quadratic form $C(\mathbf{f})=\mathbf{f}^{T} \mathbf{A} \mathbf{f}$ is convex in $\mathbf{f}$, where $\mathbf{A}$ is a symmetric and positive-definite matrix. Considering the points $\mathbf{f}_{i}, i \in[1, \ldots, n]$, the following lemma demonstrates that $C$ is convex by showing that $C^{\prime} \leq C^{\prime \prime}$ where

$$
\begin{gathered}
C^{\prime}=\left(\sum_{i=1}^{n} \xi_{i} \mathbf{f}_{i}^{T}\right) \mathbf{A}\left(\sum_{j=1}^{n} \xi_{j} \mathbf{f}_{j}^{T}\right) \text { and } \\
C^{\prime \prime}=\sum_{i=1}^{n} \xi_{i} \mathbf{f}_{i}^{T} \mathbf{A} \mathbf{f}_{i}
\end{gathered}
$$

with $\sum_{i=1}^{n} \xi_{i}=1$ and $\xi_{i} \geq 0$ for all $i \in[1, \ldots, n]$.

Matrix A is positive-definite, so

$$
\begin{gathered}
\left(\mathbf{f}_{i}-\mathbf{f}_{j}\right)^{T} \mathbf{A}\left(\mathbf{f}_{i}-\mathbf{f}_{j}\right) \geq 0 \\
\xi_{i} \xi_{j}\left(\mathbf{f}_{i}-\mathbf{f}_{j}\right)^{T} \mathbf{A}\left(\mathbf{f}_{i}-\mathbf{f}_{j}\right) \geq 0 \\
\sum_{i=1}^{n} \sum_{j=1}^{n} \xi_{i} \xi_{j}\left(\mathbf{f}_{i}-\mathbf{f}_{j}\right)^{T} \mathbf{A}\left(\mathbf{f}_{i}-\mathbf{f}_{j}\right) \geq 0 \\
\sum_{i=1}^{n} \sum_{j=1}^{n} \xi_{i} \xi_{j}\left(\mathbf{f}_{i}^{T} \mathbf{A} \mathbf{f}_{j}+\mathbf{f}_{j}^{T} \mathbf{A} \mathbf{f}_{i}\right) \leq \\
\sum_{i=1}^{n} \sum_{j=1}^{n} \xi_{i} \xi_{j}\left(\mathbf{f}_{i}^{T} \mathbf{A} \mathbf{f}_{i}+\mathbf{f}_{j}^{T} \mathbf{A} \mathbf{f}_{j}\right)
\end{gathered}
$$

Since $\sum_{i=1}^{n} \xi_{i}=1$ and $\mathbf{A}$ is symmetric

$\sum_{i=1}^{n} \sum_{j=1}^{n} \xi_{i} \xi_{j} \mathbf{f}_{i}^{T} \mathbf{A} \mathbf{f}_{j} \leq \sum_{i=1}^{n} \xi_{i} \mathbf{f}_{i}^{T} \mathbf{A} \mathbf{f}_{i}$ 
what proves that $C^{\prime} \leq C^{\prime \prime}$.

As a second lemma, consider $n$ numbers $a_{1}$, $a_{2}, \ldots, \quad a_{n}$. The maximum value of $f=\sum_{i=1}^{n} \xi_{i} a_{i}^{2}$ where $\sum_{i=1}^{n} \xi_{i}=1$ and $\xi_{i} \geq 0$ for all $i \in[1, \ldots, n]$ is $f_{M}=a_{M}^{2}, a_{M}^{2}$ being the maximum of all $a_{i}^{2}$ for $i \in[1, \ldots, n]$. Since $a_{M}^{2}$ is the maximum of all $a_{i}^{2}$ then

$$
\begin{gathered}
\left(a_{M}^{2}-a_{i}^{2}\right) \geq 0 \\
\sum_{i=1}^{n} a_{M}^{2} \xi_{i} \geq \sum_{i=1}^{n} a_{i}^{2} \xi_{i}=f \\
a_{M}^{2} \sum_{i=1}^{n} \xi_{i}=a_{M}^{2} \geq \sum_{i=1}^{n} a_{i}^{2} \xi_{i}=f
\end{gathered}
$$

Thus, $f \leq a_{M}^{2}$. The equality $f=a_{M}^{2}$ holds only when $\xi_{M}=1$ and $\xi_{i}=0$ for $i \in[1, \ldots$, $M-1, M+1, \ldots, n]$.

Combining both lemmas it is concluded that, given $l$ load cases $\mathbf{f}_{1}, \mathbf{f}_{2}, \ldots, \mathbf{f}_{l}$ and all the load cases resulting from their convex combination, the maximum of $C(\mathbf{f})=\mathbf{f}^{T} \mathbf{A} \mathbf{f}(\mathrm{a}$ convex function) is necessarily associated with the load case $M$ that yields the maximum $C\left(\mathbf{f}_{M}\right)=\mathbf{f}_{M}^{T} \cdot \mathbf{A . \mathbf { f } _ { M }}$.

\section{THE COMPLIANCE OPTIMIZATION STRATEGY}

The minimax problem presented on equation (7) gives the optimum design of a structure subjected to a loading set. The expression for the compliance, in a linear static finite element analysis, is $C(\mathbf{f})=\mathbf{f}^{T} \mathbf{K}^{-1} \mathbf{f}$, where $\mathbf{f}$ is the load vector and $\mathbf{K}$ is the stiffness matrix that is always symmetric and positivedefinite.

In order to solve the objective function $\phi$ in equation (7) it is not necessary to compute all load cases, but only those that belong to the vertices of the convex load set, i.e., the convex hull, what considerably simplifies the computational effort.

Figure 2 shows a sketch where the compliance surface and the admissible load space. It can be seen that $C=0$ occurs only if $\mathbf{f}=\mathbf{0}$. The points on the $f_{i} f_{j}$ plane (black and blue dots) are eligible load cases and the dashed polygonal line connects the points (blue dots) belonging to the convex hull of the load space. According to the previous discussion the worst compliance must be associated with one of the points in the convex hull (blue dots). Hence, only those points must be assessed in order to find the worst compliance of the entire admissible load space.

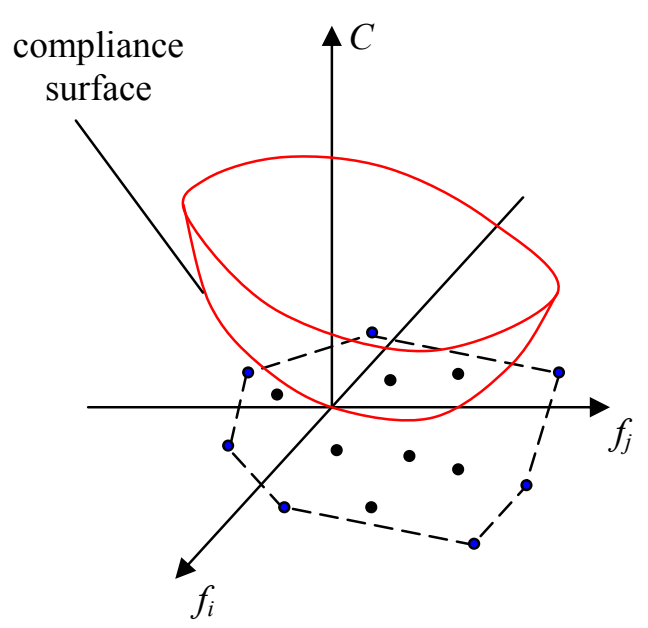

Figure 2: Compliance surface and admissible load space

\section{THE BEAM EXAMPLE}

An Euler-Bernoulli beam will be optimized for the maximum compliance due to uncertain loads. The beam has rectangular cross-section, variable thickness (height) and constant width. To avoid unrealistic zero thickness, a base beam thickness is preliminarily stated. The optimization thickness distribution will add top and bottom extra height. Figure 3 shows a sketch of the described beam only for visualization.

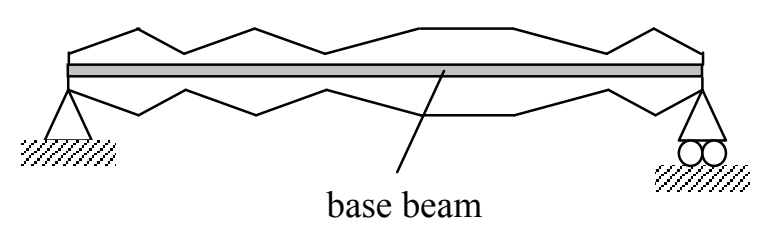

Figure 3: Variable thick beam

The beam model will be obtained using the finite element method (FEM). Each element 
may exhibit a linear thickness variation, so the whole beam has piecewise linear thickness.

The admissible load space will be represented by a collection of piecewise linear basis functions defined at specific locations along the beam. An arbitrary transverse piecewise linear loading applied to the beam is shown in Fig. 4a. Notice that the definition of more of basis functions expands the admissible load space. The piecewise linear basis functions shown in Fig. 4b are defined at five points: 1, 2, 3, 4 and 5. A particular set 1, 2, 3, 4 and 5 (the heights of the triangles in Fig. $4 \mathrm{~b}$ reflects the loading in Fig. 4a. Points 1, 2, 3 ,4 and 5 may not coincide with nodes of the finite element mesh. However, coincidence as in Fig. $4 \mathrm{a}$ is desirable in order to facilitate numerical procedures.

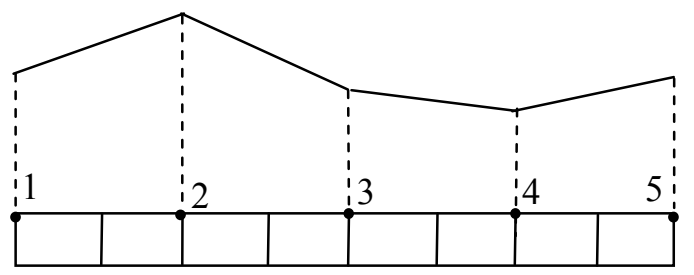

(a)

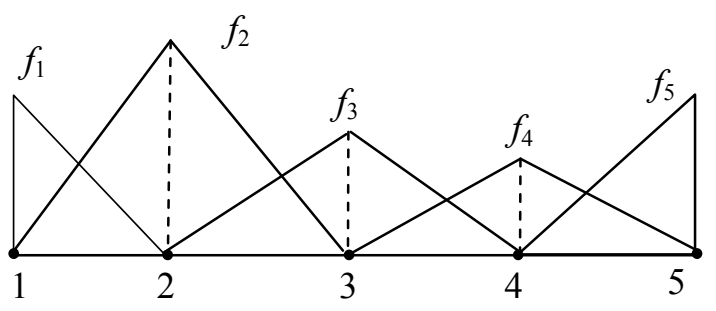

(b)

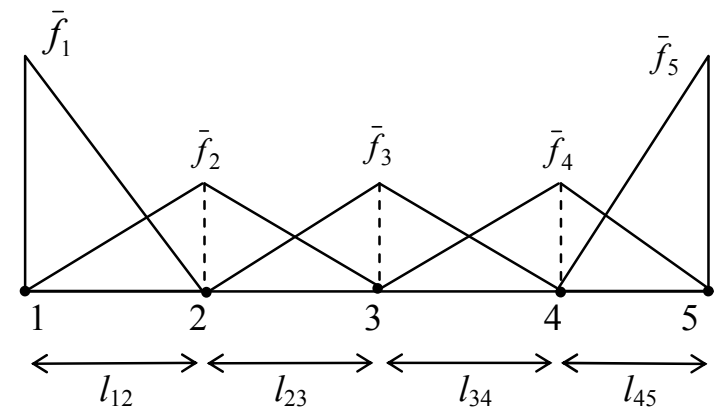

(c)

Figure 4: Discretization of the loading distribution

The loading distribution is actually described by the heights of triangles $f_{1}, f_{2}, f_{3}, f_{4}$ and $f_{5}$. If the height of triangle $i$ is denoted by $f_{i}$ it can be written as in equation (8).

$$
f_{i}=R_{i} \overline{f_{i}}
$$

where $\overline{f_{i}}$ is a scaling factor and $0 \leq R_{i} \leq 1$ is the

proportionality parameter that provides a measure of the contribution of each piecewise linear function to the applied loading. $\overline{f_{i}}$ is selected such that the areas of all triangles in Fig. $4 \mathrm{c}$ are equal to some value $\bar{f} L$ as in equation (9) where $m$ is the number of basis functions and $L$ is the beam length.

$$
\begin{aligned}
& \bar{f}_{1}=\frac{2 \bar{f} L}{l_{12}} \\
& \bar{f}_{i}=\frac{2 \bar{f} L}{l_{i-1, i}+l_{i, i+1}} \text { for } 1<i<m \\
& \bar{f}_{m}=\frac{2 \bar{f} L}{l_{m-1, m}}
\end{aligned}
$$

$R_{i}$ defines the contribution of each base function. In order to maintain the same resulting load $\bar{f} L$, the summation of all $R_{i}$ 's must be as stated in equation (10).

$$
\sum_{i=1}^{m} R_{i}=1
$$

The distributed loadings represented by piecewise linear basis functions may by applied as a convex combination according to equation (10) or individually. Figure $4 \mathrm{~b}$ shows a case where the summation of the contribution of the all individual basis loading is equal to the resultant of the specified load of Fig. 4a. For the distribution $i$ the resulting force $F_{i}$ is given by:

$$
F_{i}=R_{i} \overline{f_{i}} L
$$

The min-max technique employed gives the worst loading distribution in terms of $R_{i}$. Despite the fact that $\bar{f}$ is arbitrary, it is made equal do $1 / L$ for the numerical simulations in this section.

There is another constraint, besides the one stated in equation (10), related to the beam 
constant volume. Equation (12) states the second constraint in terms of thickness.

$$
\frac{h_{1}}{2}+\sum_{i=2}^{n-1} h_{i}+\frac{h_{n}}{2}=(n-1) h
$$

where $n$ is the number of nodes and $h$ is the corresponding beam thickness, in case of constant thickness of the entire beam. Equations (10) and (12) impose bounds to the optimization problem stated in equation (7).

Solution of the optimization problem initiates by assessing 10,000 random designs in order to reduce the risk of convergence to local optima. The best random point is chosen to start the Powell's search [5]. Advantages of the Powell's method are its simplicity and its independence of gradient information. This is particularly necessary in this application since function $\phi$ defined in equation (7) may not be smooth with respect to $\mathbf{h}$. The optimization stops when the relative difference between the previous and present values of $\phi$ does not exceed 0.001 .

As an example a beam with different number of nodes and different number of basis functions is optimized for maximum compliance. The beam material is aluminum with Young modulus of $70 \mathrm{GPa}$ and the beam length is $0.3 \mathrm{~m}$. Also, the beam is simply supported at both ends. Figure 4 shows an example of the beam discretization, where the number of nodes can vary considering that all elements must have the same length for the entire beam. The equivalent uniformly distributed loading $\bar{f}$ presented in equation (9) is chosen to give a unit resultant force $\bar{f} L$ $=1 \mathrm{~N}$.

\section{RESULTS AND CONCLUSIONS}

Depending on the mesh refinement different number of load cases may be used. Table 1 shows the minimum compliances for a beam with base thickness of $h_{\text {base }}=0.5 \mathrm{~mm}$ obtained when $n=9$ and $h=1.5 \mathrm{~mm}$ in equation (12). Columnwise it can be seen that more nodes imply more flexibility to sustain the uncertain load cases. If the number of nodes is fixed more load cases always leads to higher compliances. This is because the load space is broader what increases the chances of causing damage to the structure.

\begin{tabular}{cccccc}
\hline \multirow{2}{*}{$\begin{array}{c}\text { number } \\
\text { of nodes }\end{array}$} & \multicolumn{5}{c}{ number of load cases } \\
\cline { 2 - 6 } & 2 & 3 & 5 & 7 & 9 \\
\hline 3 & 3.716 & 5.486 & - & - & - \\
5 & 3.599 & 5.291 & 6.752 & - & - \\
7 & 3.569 & 5.253 & - & 6.988 & - \\
9 & 3.559 & 5.241 & 6.712 & - & 7.069 \\
\hline
\end{tabular}

Table 1: Minimum beam compliances $(\mu \mathrm{J})$

In Table 1 the base beam thickness $h_{\text {base }}$ and $h$ were fixed. However, variation of both of them certainly affects the optimal design. If one maintains the sum $h+h_{\text {base }}$ constant a greater $h$ means more flexibility to the structure so it can adapt to withstand broader load spaces while a greater base beam thickness implies that the thickness distribution cannot vary as much what renders the structure vulnerable to loading variations. Table 2 shows the effects of the balance between $h$ and $h_{\text {base }}$ in the optimal compliance when 9 nodes are considered along with 5 load cases. It is clear from Table 2 that fixing a design configuration is a bad strategy when there are multiple potential load cases.

\begin{tabular}{ccc}
\hline$h_{\text {base }}(\mathrm{mm})$ & $h(\mathrm{~mm})$ & compliance $(\mu \mathrm{J})$ \\
\hline 0.0 & 2.0 & 6.702 \\
0.5 & 1.5 & 6.712 \\
1.0 & 1.0 & 7.022 \\
1.5 & 0.5 & 7.673 \\
2.0 & 0.0 & 10.77 \\
\hline
\end{tabular}

Table 2: Effect of thickness variability

Making $h+h_{\text {base }}$ constant is equivalent to admitting that a fixed amount of structural mass is available for the optimization. When $h=0$ the thickness distribution is perfectly uniform and there is no room for redistribution of the available material in order to better withstand the loading space. Figure 5 shows the thickness distribution of the optimal designs. 


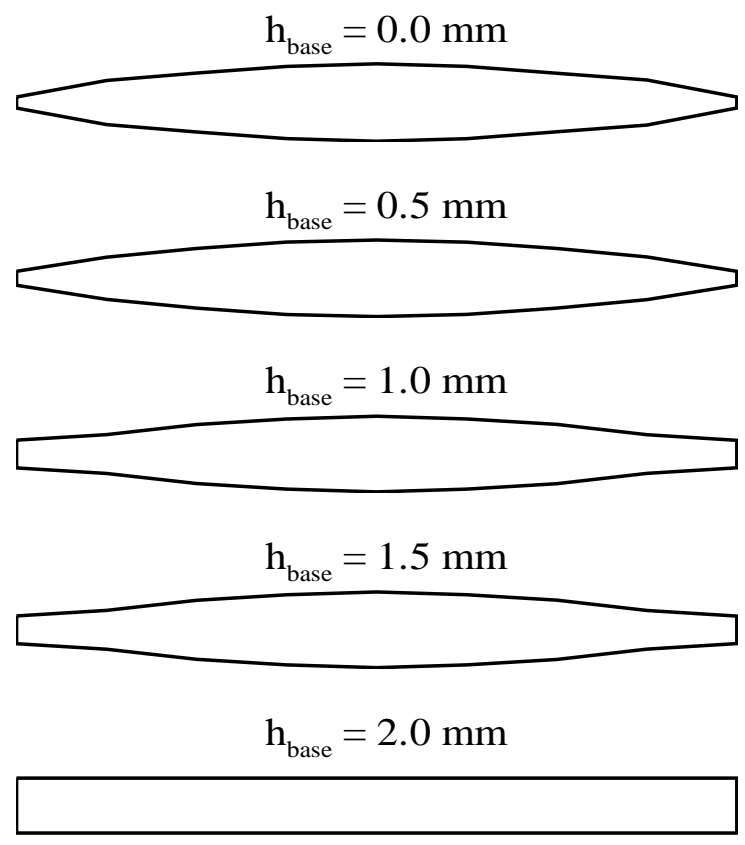

Figure 5: Optimal beams

A new technique has been proposed for compliance minimization of structural components subjected to many loading combinations. In real applications it is not unusual to have structures that experience many different load cases during operation. In particular, structural components for aerospace applications must withstand severe loads while operating in hostile environmental conditions. For example, a vertical control surface (rudder) of an aircraft is subjected to approximately 100 important load cases, including but not limited to maneuver loads, gust loads and landing loads. When an airplane rudder is optimized for minimum weight, maximum strain constraints are frequently active; either the spars or the panels could fail due to large stresses. Therefore, minimizing compliance becomes an important factor in the design of efficient, lightweight control surfaces.

\section{REFERENCES}

[1] I. Das, and J. E. Dennis (1998) Normalboundary intersection: a new method for generating the Pareto surface in nonlinear multicriteria optimization problems. SIAM Journal of Optimization, 8(3): 631-657.

[2] A. Cherkaev, and E. Cherkaeva (1999) Stable optimal design for uncertain loading conditions, Homogenization, World Scientific, edited by V. Berdichevsky, pp. 193-213.
[3] V. B. Hammer, M. P. Bendsøe, R. Lipton, and P. Pedersen (1997) Parametrization in laminate design for optimal compliance. International Journal of Solids and Structures, 34(4): 415-434.

[4] P. Pedersen (1991) On thickness and orientational design with orthotropic materials. Structural and Multidisciplinary Optimization Journal, 3: 69-78.

[5] M. J. D. Powell (1964) An efficient method for finding the minimum of a function of several variables without calculating derivatives. Computers Journal, 7: $155-162$. 